\title{
Genetic Mutations in CFTR Protein Gene Cause Cystic Fibrosis- Its Symptoms, Treatment, and Incidence in Pakistan
}

\author{
Dr. Muhammad Farooq Sabar, Fatima Arshad
}

Centre for Applied Molecular Biology, University of the Punjab, Lahore, Pakistan

*Corresponding author: Fatima Arshad

\section{Abstract}

Genetic mutations in the CFTR protein gene cause Cystic Fibrosis. For disease occurrence, individuals have to inherit two defective genes of CFTR from each parent. CFTR mutations are distributed into six classes. Though CF affects all ethnic as well as racial groups, but in Caucasians of North European origin, it is more common. The clinical representations of $\mathrm{CF}$ include raised $\mathrm{NaCl}$ cons. in sweat glands, deficiency of pancreatic enzymes, azoospermia in adult males, and decline in lung function which leads to pulmonary exacerbations. The therapies for CF are gene therapy, CFTR modulators, potentiators, correctors, and stop codon suppressors. The incidence of CF in Pakistan is low as it is more common in Caucasians. Due to lack of suitable diagnostic tools, this disease is often neglected in Pakistani population.

Keywords: Cystic Fibrosis, ${ }^{*}$ CFTR protein gene, mutations, clinical manifestations, incidence *Cystic Fibrosis Transmembrane Conductance Protein.

Copyright @ 2019: This is an open-access article distributed under the terms of the Creative Commons Attribution license which permits unrestricted use, distribution, and reproduction in any medium for non-commercial use (NonCommercial, or CC-BY-NC) provided the original author and source are credited.

\section{INTRODUCTION}

In the late 1930s, Cystic Fibrosis was first documented as a disease. This term was derived from its "cyst formation" and "scarring" characteristics in the patient's pancreas [1]. Based on the autosomal recessive inheritance pattern of disease, it is speculated that $\mathrm{CF}$ must be triggered by the single gene fault [2].

CF is caused by CFTR gene mutations that cause defective CFTR protein [3-5]. On the surface of epithelial membrane, CF Transmembrane Conductance Proteins are located that act as chloride channels which in turn control the epithelial sodium channel as well as different other anionic channels present on the surface of the cell. This intricate relationship of channels controls the electrochemical gradient which lets proper airway, surface liquid depth, and mucus viscosity $[6,7]$. With the defect or missing of these proteins, the body produces viscous and thick mucus [8].

Above 1,800 CFTR gene mutations have been identified till now (Patient Registry: Annual Data, 2012). Upon the disease causing mechanism, CFTR mutations are distributed into six classes (Table 1). Though CF affects all ethnic as well as racial groups, but in Caucasians of North European origin, it is more common [3].
Before, there were no such medical therapies that could aim the basic genetic cause of this disease and could address its signs/ symptoms. Like, therapies for airway clearance have been used to remove mucus from air ways on a daily basis, to help in food digestion pancreatic enzyme replacement has been taken with every meal, and to treat lung infection antibiotics are used [9]. So, wide research is being done for the development of such agents that could affect $\mathrm{CF}$ on a genetic level[10]. The research upon CFTR modifier medications epitomizes a significant revolution in treating $\mathrm{CF}$. the reason is, such agents directly target the basic defect of $\mathrm{CF}$ rather than targeting the effects caused by the disease. Though the first FDA-approved CFTR modifier is Ivafactor and other medicines are in progress [11].

\section{Genetics of CFTR}

Cystic Fibrosis being a monogenic disorder affects mainly the Caucasian descent individuals. For disease occurrence, individuals have to inherit two defective genes of CFTR from each parent [12]. The distribution of the CFTR gene results in protein (CFTR) expression. Its expression is responsible for the transportation of chloride and other anions in and out of the cell. The location of the CFTR gene is at the long arm of chromosome 7 [8]. There are around 1,900 
CFTR mutations that have been reported up to now. Of them only twenty mutations have a frequency of $0.1 \%$. The most common variant is F508del having 90\% allelic frequency [13].

\section{Structure of CFTR protein}

CFTR protein is made up of 1,480 amino acids having cyclic adenosine monophosphate controlled chloride ion channel [1]. The channel is an ATPbinding transporter having 5 domains. Of them, two are transmembrane domains that are responsible for formation of channel pore, the other one is regulatory domain and other two are nucleotide binding domains [14].

\section{Synthesis of CFTR protein}

The CFTR gene expression is initiated by extracellular signals. The gene transcription leads to the synthesis of mRNA that migrates towards nuclear pores to interact with ribosomes [15]. With the arrangement of transfer RNA, the translation of the CFTR gene into amino acid chains occurs. The resulted polypeptide chains are gathered as the immature product of CFTR protein and further folded within the ER lipid bilayer. The other spacking into Golgi vesicles occurs [16]. At last, for the final expression upon the epithelial cells, the channel is transferred to the cell surface [17].

\section{Mutation Classification}

CFTR gene mutations have their impact on every step involved in the synthesis of protein. The steps are gene transcription, protein translation, folding, transfer, expression as wells as gating of the channel on the surface of cells [17].

Table-1: Classification of Genetic Mutations [9, 18, 19]

\begin{tabular}{|l|l|l|}
\hline Class & Mutation & Description \\
\hline I & G542X & $\begin{array}{l}\text { Synthesis of CFTR protein is defective due to PTCs- Premature } \\
\text { Termination Codon }\end{array}$ \\
\hline II & F508del & $\begin{array}{l}\text { Compromised processing: misfolding of protein CFTR; } \\
\text { maturation of defective protein; degradation of premature protein; } \\
\text { proteins can't reach towards the apical surface of cells }\end{array}$ \\
\hline III & G551D & $\begin{array}{l}\text { Improper regulation: reaching of CFTR proteins towards the } \\
\text { apical surface, but inactivated by Camp or ATP; exhibition of } \\
\text { abnormal chloride channel gating }\end{array}$ \\
\hline IV & R334W & $\begin{array}{l}\text { Inappropriate function: reduction in the transport of chloride ions } \\
\text { while full length proteins reach apical surface }\end{array}$ \\
\hline V & R117H & $\begin{array}{l}\text { Due to abnormalities in splicing and promoter, synthesis plus an } \\
\text { expression of CFTR got reduced }\end{array}$ \\
\hline V1 & $1811+1.6 \mathrm{kbA}>\mathrm{G}$ & $\begin{array}{l}\text { CFTR goes to the apical surface of the cell, but mutations in C } \\
\text { terminus because accelerated turnover. }\end{array}$ \\
\hline
\end{tabular}

\section{Clinical manifestation of CF}

\section{Sweat Glands}

One of the representations of $\mathrm{CF}$ is the excess of secretion of sodium chloride through sweat glands. In the absorptive duct of sweat gland, the reabsorption of $\mathrm{Cl}$ is regulated by CFTR protein. This process is hindered by the absence of CFTR and in case of CF, promotes hypertonic beads of sweat [20]. The normal concentration of sweat chloride is $20 \mathrm{mmol} / \mathrm{L}$. The carriers, that have single mutation, show a minor rise in the levels of sweat chloride, like mean value is $26 \mathrm{mmol} / \mathrm{L}$. In the patients that have one severe and other mild mutation, the average sweat chloride concentration value is of $80 \mathrm{mmol} / \mathrm{L}$. However, with two severe mutations, the concentration becomes largely elevated up to $100 \mathrm{mmol} / \mathrm{L}$. That would demonstrate a relationship between CFTR functionality and sweat chloride levels [8].

\section{Pancreas and GI tract}

The deficiency of pancreatic enzymes is expected to be exist in $90-95 \%$ of patients. The improper digestive enzyme secretions along with fat malabsorption in the GI tract shows severe consequences like steatorrhea. The likelihood of gall stones and Hepatobiliary disease would become greater due to ionic imbalance in the biliary tract. The pancreatic abnormalities might also cause CF related diabetes, having the occurrence of $25 \%$ in the patients with CF of age 25 years [21].

\section{Reproductive system}

Azoospermia can be observed in $\mathrm{CF}$ male patients. In $99 \%$ of $\mathrm{CF}$ adult males, there is inherited bilateral absence of vas deferens that would block the spermatozoa transport from testes to the vas deferens and cause azoospermia. In general, CF females have a less severe outcome of infertility as compared to males [16].

\footnotetext{
Airways

Lung and airways are the key clinical indices in CF. The protein expression occurs in submucosal glands and on ciliated epithelial cell apical surfaces in bronchial tissues [21]. Due to the dysfunction or absence of CFTR protein, less $\mathrm{Cl}$ (chloride) efflux and uncontrolled hyperactive absorption of $\mathrm{Na}$ (sodium
} 
ions) occurs. The ASL dehydration is caused by imbalance in osmotic gradient that is responsible for increased mucus and reduced mucociliarytransport. This situation regularly depreciates chronic local inflammation, bronchiectasis, plugging of small airways and bacterial infection without interference.

Moreover, increased deterioration of lung function in cystic Fibrosis is contributed by repeated pulmonary exacerbations [20].

\section{Disease modifying therapies for Cystic Fibrosis}

These approaches can divided into two categories: First is gene therapy that involves the agents which aim at the gene mutation and second is CFTR modulators that address the linked protein defect.

\section{Gene therapy}

This has the involvement of delivery methods via viral and non-viral vectors. As these targets the delivering copies of normal CFTR DNA coding sequence to target cells. The delivery of therapeutic DNA is done via viral recombinant vector to the host cell. The normal coding sequence of CFTR is transcribed into mRNA; otherwise it becomes integrated into the genome of host cell [22].

In case of non-viral gene therapy, vectors other than viral vectors deliver the naked DNA into the cell. For a very long time, gene therapy research studies for $\mathrm{CF}$ have not been providing vibrant evidences to relate the findings of this study for the benefit of clinical practice [22]. The new research about gene therapy is now limited mainly because of the sign that transfer of genes is way more challenging than previously thought.

\section{CFTR modulators}

The small molecules for managing the $\mathrm{CF}$ have come in the rule. They are called CFTR modulators. The research depends upon the facts about classes of CFTR mutations plus gives emphasis on directing exact molecules to certain mutations. Such specialized molecules are a typical example of modified medicine [23]. The classification of CFTR modulators comprises of three classes of agents. They are stop codon suppressors, potentiators, and correctors. The automated approach helps in their identification by permitting the rapid screening of modulators [23].

\section{Stop codon suppressors}

Among all CFTR mutations, 5-10\% explains about the nonsense or premature stop mutations by having regional variation. As a result the CFTR protein is produced that would then be transferred to the surface of the cell. A few examples of read-through agents are there. Gentamicin is the most investigated antibiotic in this regard. Its purpose is to incorporate an amino acid instead of stop-codon to continue the normal translation [16]. To assess the efficiency of Gentamicin, a study was conducted in 24 patients of CF. For CFTR stop mutations, out of 19 patients, some were homozygous while others were heterozygous. Besides, $5 \mathrm{CF}$ patients were homozygous for F508del class II mutation. In patients with the stop mutations, Gentamicin significantly reduced the nasal potential difference while there was no considerable difference could be seen in the patients having the F508del mutation. Another example of a read- through agent is Ataluren which is previously known as PTC124. It has significance over gentamicin being a 4-15 fold stronger agent. The initial research was conducted in healthy adults and it showed no increase in safety alarms. In addition, investigations of mononuclear peripheral blood cells presented common CFTR protein elongation. This could have happened because of ribosomal nonspecific stop codons. It was concluded that Ataluren induce functional CFTR production [24].

\section{CFTR correctors}

The intention of CFTR correctors is the enhancement of the area of CFTR functional protein for transportation towards the surface of the cell. The target of correctors is used to target F508del Class II mutations. One corrector molecule, VX-809, can be used as treating agent for CF patients. The studies (invitro) of VX-809 have verified it as an effective and astute CFTR corrector that is preferable for extension of clinical researches [17]. For the adults who are F508del CFTR mutation homozygous, VX-809 is considered efficient. Overall, there are no noteworthy differences of lung function between control and experimental trials [17].

Another oral CFTR corrector is VX-661. It is similar to Lumafactor and has been developed by Vertex Pharmaceuticals for treatment of CF. In vitro, the greater activity was noticed combining VX-661 and ivacaftor as compared to VX-661 alone (Vertex Pharmaceuticals, 2013). The primary results show that VX-661 could be a favorable corrector that might help CF patients after more research (National Institute of Health, 2013).

\section{CFTR potentiators}

For CFTR channels having Class III and Class IV mutations (gating\& conductance), CFTR potentiators raise the open possibility. Plus, they can also increase the open likelihood of CFTR channels having Class II of mutations for example F508del [25]. For class III mutations like G551D, Ivacaftor is a CFTR potentiator (formerly known as VX-770).

For the treatment of $\mathrm{CF}$ patients, a trial was conducted in the year 2012 on January 31, using ivacaftor (Vertex Pharmaceuticals). CF patients of 6 years old and above with the Class III G551D mutation were studied (Patient Registery, 2012 and Food \& Drug Administration, 2013). It enhanced the time for the CFTR channel to be opened, allowing chloride ions to move into CFTR proteins present on the epithelial cell 
surface (Vertex Pharmaceuticals, 2013 and [26]. Ivacaftor targets the basic fault in CF. The dosage approved is one tablet of $150-\mathrm{mg}$ of ivafactor orally taken every 12 hours with fat containing foods (Vertex Pharmaceuticals, 2013).

\section{CF incidence in Pakistan}

The mutations in CFTR differ with respect to genetic background. Due to lackof suitable diagnostic tools, this disease is often neglected in Pakistani population. For the actual diagnosis of $\mathrm{CF}$ in a population, mutations' identification along with frequencies is important to design gene probes. With the changing severity of the disease different mutations are associated. This information becomes significantly relevant in a country like Pakistan having a diverse ethnic population [27] studied the CF patients in Aga Khan University. The primary data of the study, involving $15 \mathrm{CF}$ patients, showed $60 \%$ frequency for DF508 and $33 \%$ frequency for patients that were homozygous for F508del mutation [28].

The other frequencies within the range of 30$54 \%$ have been reported by different authors in Pakistani children [29-33]. Though, Frossard et al. found a chance to study Pakistanis in UAE. They reported $86 \%$ frequency of DF508 mutation in Pakistanis having Baluchi origin and assumed that early Baluchistan might have been the foundation of this mutation. They further suggested about the geographic distribution of mutations that they might be helpful in origin and migration studies of populations someday [34].

Shah et al. recruited patients from Aga Khan University, Pakistan with clinically suspected Cystic Fibrosis and raised sweat chloride values. By allele specific PCR, different mutations like S549R, DF508, Y569D, S549N, G553X, G551X and G551D were screened. With direct DNA sequencing, 10, 11, and 12 CFTR exons were sequenced. Out of $150 \mathrm{CF}$ patients, $26(17.3 \%)$ showed DF508 mutation tested by PCR. Out of them one person was an F508S549N compound heterozygote. The step towards defining mutations present in Pakistanis' CF suggested that mutation DF508 is rare while S549 was the only added mutation that was identified in 10, 11, 12 CFTR exons [35].

Identification as well as the frequency of other left mutations is obligatory to formulate appropriate and well-suited tests for better understanding and managing the disease.

\section{Declaration of Interest}

The authors of this article report no conflict of interest.

\section{REFERENCES}

1. Andersen, D. H. (1938). Cystic fibrosis of the pancreas and its relation to celiac disease: a clinical and pathologic study. American journal of Diseases of Children, 56(2), 344-399.

2. Kerem, E., Corey, M., Kerem, B. S., Rommens, J., Markiewicz, D., Levison, H., \& Durie, P. (1990). The relation between genotype and phenotype in cystic fibrosis - analysis of the most common mutation ( $\Delta \mathrm{F} 508)$. New England Journal of Medicine, 323(22), 1517-1522.

3. Mohon, R. T., Wagener, J. S., Abman, S. H., Selfzer, W. K., \& Accurso, F. J. (1993). Relationship of genotype to early pulmonary function in infants with cystic fibrosis identified through neonatal screening. The Journal of pediatrics, 122(4), 550-555.

4. Welsh, M. J., \& Smith, A. E. (1993). Molecular mechanisms of CFTR chloride channel dysfunction in cystic fibrosis. Cell, 73(7), 1251-1254.

5. Bear, C. E., Li, C., Kartner, N., Bridges, R. J., Jensen, T. J., Ramjeesingh, M., \& Riordan, J. R. (1992). Purification and functional reconstitution of the cystic fibrosis transmembrane conductance regulator (CFTR). Cell, 68(4), 809-818.

6. Rich, D. P., Anderson, M. P., Gregory, R. J., Cheng, S. H., Paul, S., Jefferson, D. M., ... \& Welsh, M. J. (1990). Expression of cystic fibrosis transmembrane conductance regulator corrects defective chloride channel regulation in cystic fibrosis airway epithelial cells. Nature, 347(6291), 358.

7. Rowe, S. M., Accurso, F., \& Clancy, J. P. (2007). Detection of cystic fibrosis transmembrane conductance regulator activity in early-phase clinical trials. Proceedings of the American Thoracic Society, 4(4), 387-398.

8. O'Sullivan, B.P., \& S.D. (2009). Freedman, Cystic fibrosis. Lancet, 373(9678): 1891-904.

9. Anderson, P. (2010). Emerging therapies in cystic fibrosis. Therapeutic advances in respiratory disease, 4(3), 177-185.

10. Pettit, R. S., \& Fellner, C. (2014). CFTR modulators for the treatment of cystic fibrosis. Pharmacy and Therapeutics, 39(7), 500.

11. Kirk, K. L., \& Wang, W. (2011). A unified view of cystic fibrosis transmembrane conductance regulator (CFTR) gating: combining the allosterism of a ligand-gated channel with the enzymatic activity of an ATP-binding cassette (ABC) transporter. Journal of Biological Chemistry, 286(15), 12813-12819.

12. Burney, T. J., \& Davies, J. C. (2012). Gene therapy for the treatment of cystic fibrosis. The application of clinical genetics, 5, 29.

13. Rosenecker, J., Huth, S., \& Rudolph, C. (2006). Gene therapy for cystic fibrosis lung disease: current status and future perspectives. Current opinion in molecular therapeutics, 8(5), 439.

14. Rommens, J. M., Iannuzzi, M. C., Kerem, B. S., Drumm, M. L., Melmer, G., Dean, M., ... \& Hidaka, N. (1989). Identification of the cystic 
fibrosis gene: chromosome walking and jumping. Science, 245(4922), 1059-1065.

15. Davis, P., Drumm, M., \& Konstan, M.W.(1996) Cystic fibrosis. Am J Respir Crit Care Med, 154: 1229-1256.

16. Clancy, J. P., \& Jain, M. (2012). Personalized medicine in cystic fibrosis: dawning of a new era. American journal of respiratory and critical care medicine, 186(7), 593-597.

17. Grasemann H, Ratjen F. Emerging therapies for cystic fibrosis lung disease. Expert opinion on emerging drugs. 2010 Dec 1;15(4):653-9.

18. Rowe, S., Miller S, Sorscher, E.J. (2005). Cystic fibrosis. N Engl J Med, 352: 1992-2001.

19. Flume, P. A., \& Van Devanter, D. R. (2012). State of progress in treating cystic fibrosis respiratory disease. BMC medicine, 10(1), 88.

20. Derichs, N., Jin, B. J., Song, Y., Finkbeiner, W. E., \& Verkman, A. S. (2011). Hyperviscous airway periciliary and mucous liquid layers in cystic fibrosis measured by confocal fluorescence photobleaching. The FASEB Journal, 25(7), 23252332.

21. Griesenbach, U., \& WFW Alton, E. (2012). Progress in gene and cell therapy for cystic fibrosis lung disease. Current pharmaceutical design, 18(5), 642-662.

22. Pettit, R. S. (2012). Cystic fibrosis transmembrane conductance regulator-modifying medications: the future of cystic fibrosis treatment. Annals of Pharmacotherapy, 46(7-8), 1065-1075.

23. Pettit, R. S. (2012). Cystic fibrosis transmembrane conductance regulator-modifying medications: the future of cystic fibrosis treatment. Annals of Pharmacotherapy, 46(7-8), 1065-1075.

24. Pedemonte, N., Lukacs, G. L., Du, K., Caci, E., Zegarra-Moran, O., Galietta, L. J., \& Verkman, A. S. (2005). Small-molecule correctors of defective $\triangle$ F508-CFTR cellular processing identified by high-throughput screening. The Journal of clinical investigation, 115(9), 2564-2571.

25. Van Goor, F., Hadida, S., Grootenhuis, P. D., Burton, B., Stack, J. H., Straley, K. S., ... \& Wine, J. J. (2011). Correction of the F508del-CFTR protein processing defect in vitro by the investigational drug VX-809. Proceedings of the
National Academy of Sciences, 108(46), 18843 18848.

26. Bhutta, Z. A. (2000). Why has so little changed in maternal and child health in south Asia?. Bmj, 321(7264), 809-812.

27. Bhutta, Z. A., Moattar, T., \& Shah, U. (2000). Genetic analysis of cystic fibrosis in Pakistan: a preliminary report. J Pak Med Assoc, 50(7), 217219.

28. Bowler, I. M., Estlin, E. J., \& Littlewood, J. M. (1993). Cystic fibrosis in Asians. Archives of disease in childhood, 68(1), 120-122.

29. Malone, G., Haworth, A., Schwarz, M. J., Cuppens, H., \& Super, M. (1998). Detection of five novel mutations of the cystic fibrosis transmembrane regulator (CFTR) gene in Pakistani patients with cystic fibrosis: Y569D, Q98X, 296+ 12 (T> C), 1161delC and 621+ 2 (T> C). Human mutation, 11(2), 152-157.

30. Romey, M. C., Guittard, C., Chazalette, J. P., Frossard, P., Dawson, K. P., Patton, M. A., \& Bozon, D. (1999). Complex allele [-102T> A+ $\mathrm{S} 549 \mathrm{R}(\mathrm{T}>\mathrm{G})$ ] is associated with milder forms of cystic fibrosis than allele S549R (T> G) alone. Human genetics, 105(1-2), 145-150.

31. Schwarz, M. J., Super, M., Wallis, C., Beighton, P., Newton, C., Heptinstall, L. E., \& Bilto, D. (1990). $\Delta$ F508 testing of the DNA bank of the Royal Manchester Children's Hospital. Human genetics, 85(4), 428-430.

32. Spencer, D. A., Venkataraman, M., Higgins, S., Stevenson, K., \& Weller, P. H. (1994). Cystic fibrosis in children from ethnic minorities in the West Midlands. Respiratory medicine, 88(9), 671675.

33. Frossard, P. M., Girodon, E., Dawson, K. P., Ghanem, N., Plassa, F., Lestringant, G. G., \& Goossens, M. (1998). Identification of cystic fibrosis mutations in the United Arab Emirates. Human mutation, 11(5), 412-413.

34. Shah, U., Frossard, P., \& Moatter, T. (2009). Cystic fibrosis: defining a disease under-diagnosed in Pakistan. Tropical Medicine \& International Health, 14(5), 542-545. 